Article

\title{
The Effects of Lung-Moistening Herbal Medicines on Bleomycin-Induced Pulmonary Fibrosis Mouse Model
}

\author{
Junmo Ahn ${ }^{1,+}{ }^{\text {, Hyejin Joo }}{ }^{2,+}{ }^{\text {, Jihye Park }}{ }^{3}$, Jae-Woo Park ${ }^{1}$, Kwan-Il Kim ${ }^{1}$, Hee-Jae Jung ${ }^{1}$, \\ Youngmin $\mathrm{Bu}^{1}$ and Beom-Joon Lee ${ }^{1, * \mathbb{D}}$ \\ 1 College of Korean Medicine, Kyung Hee University, Seoul 02447, Korea; lzyajg@nate.com (J.A.); \\ pjw2907@khu.ac.kr (J.-W.P.); myhappy78@naver.com (K.-I.K.); hanfish@khmc.or.kr (H.-J.J.); \\ ymbu@khu.ac.kr (Y.B.) \\ 2 College of Science in Korean Medicine, Graduate School, Kyung Hee University, Seoul 02447, Korea; \\ joosh561@naver.com \\ 3 Department of Biomedical Science and Technology, Graduate School, Kyung Hee University, Seoul 02447, \\ Korea; ji_hye23@naver.com \\ * Correspondence: franchisjun@naver.com \\ + These authors contributed equally as first author.
}

Received: 5 November 2019; Accepted: 9 January 2020; Published: 12 January 2020

check for updates

\begin{abstract}
In traditional medicine, lung-moistening herbal medicines (LMHM) are regarded as a major option for treating symptoms of pulmonary fibrosis (PF) including dry cough and dyspnea. As PF agents are being applied to the development of lung cancer agents, PF and lung cancer are reported to have high pathological and pharmacological relationships. This study was proposed to identify candidates for the treatment of PF via investigating the effect of LMHM on PF mouse model. PF was induced by intratracheal instillation of bleomycin. Six water extracts of LMHM such as Farfarae Flos (FAF), Trichosanthis Semen (TRS), Lilii Bulbus (LIB), Adenophorae Radix (ADR), Asteris Radix (ASR), and Scrophulariae Radix (SCR) were prepared and administered $(300 \mathrm{mg} / \mathrm{kg}$ ) orally for 10 days after induction. The changes in body weight, histopathology, and immune cell of bronchoalveolar lavage fluid (BALF) were investigated. Among those, LIB and ADR significantly decreased the deposition of collagen and septal thickness of alveolar and terminal bronchiole. Moreover, SCR, TRS, LIB, and ADR decreased total cells, macrophages, and lymphocytes in BALF. Taken together, ADR and LIB could be the candidates to reduce PF. Further studies on their effects at different doses and analysis of their underlying molecular mechanisms are needed.
\end{abstract}

Keywords: pulmonary fibrosis; bleomycin; herbal medicine; broncho alveolar lavage fluid; inflammation

\section{Introduction}

Idiopathic pulmonary fibrosis (IPF) is a chronic progressive interstitial lung disease that causes chronic respiratory distress and cough, which leads to death due to hypoxia or myocardial infarction. It has been diagnosed by histopathologic and/or radiologic pattern of usual interstitial pneumonia (UIP) without an identifiable cause of lung injury [1]. In UIP, honeycombing and reticular opacity with/without traction bronchiectasis were mainly observed in high-resolution $\mathrm{CT}$, and patchy dense fibrosis including fibroblastic foci, honeycombing changes, and normal parenchyma appeared mixed at a lower magnification of lung biopsy [2]. IPF itself is a fatal lung disease and also considered closely related to lung cancer [3]. The pathological similarity between IPF and lung cancer [4], the high prevalence rate of lung cancer in IPF patients, inducing IPF during the chemotherapy and radiation 
therapy for lung cancer patients, and the applicability of IPF agents to lung cancer treatments might be the evidence of intimate relationship in clinical applications or research $[5,6]$.

However, pathological mechanisms have not been fully established. Currently, steroids, interferon- $\gamma$, N-acetylcysteine, and platelet-derived growth factor receptor blockers have been reported to show some effect, but no drug has proven to be effective. Clinically, pirfenidone and nintedanib have also been reported to reduce the progression of the disease, but the effect of these agents is debated $[1,7,8]$.

In traditional medicine, IPF has been considered a chronic progressive disease with symptoms of dry cough and dyspnea due to lung Qi deficiency, lung Yin deficiency, lung dryness due to Yin deficiency or lung heat, blood stasis, phlegm, and dampness [9-13]. Moistening the lung, applied in treating cough caused by lung dryness, has been regarded as one of the key therapeutic approaches for symptom management of IPF [9-11].

In this study, we aimed to identify the therapeutic candidates for IPF by investigating the effects of several herbal medicines on overcoming lung dryness. We selected six medicines with moistening properties and confirmed, based on thorough literature review, that no pharmacological study on pulmonary fibrosis $(\mathrm{PF})$ has been reported yet. Aqueous extracts of samples were administered orally to a mouse model of bleomycin-induced fibrosis, which is the most popular model for IPF [14].

\section{Materials and Methods}

\subsection{Sample Preparation}

The dried raw materials of the following six herbal medicines were obtained from Kyung Hee Herb Pharm (Kangwon-do, Korea), which are the same as those prescribed to patients in Kyung Hee University medical center (Seoul, Korea): Farfarae Flos (FAF), Trichosanthis Semen (TRS), Lilii Bulbus (LIB), Adenophorae Radix (ADR), Asteris Radix et Rhizoma (ASR), and Scrophulariae Radix (SCR). Two hundred grams of each herb were extracted by boiling in $1500 \mathrm{~mL}$ water for $2 \mathrm{~h}$ at $100{ }^{\circ} \mathrm{C}$. The origin, used part, and extraction yield of each medicine are given in Table 1.

Table 1. List of herbal medicines.

\begin{tabular}{|c|c|c|c|c|c|}
\hline Botanical Name & Scientific Name & $\begin{array}{l}\text { Part of } \\
\text { Use }\end{array}$ & $\begin{array}{l}\text { Main Traditional } \\
\text { Use [15] }\end{array}$ & $\begin{array}{c}\text { Sample } \\
\text { Specimen * }\end{array}$ & Yield (\%) \\
\hline Farfarae Flos & Tussilago farfara L. & flower & $\begin{array}{l}\text { Moisten the lung to } \\
\text { suppress cough }\end{array}$ & HLL-001 & 30.3 \\
\hline Trichosanthis Semen & $\begin{array}{c}\text { Trichosanthes } \\
\text { kirilowii Maxim. }\end{array}$ & seed & $\begin{array}{l}\text { Moisten the lung and } \\
\text { resolve phlegm }\end{array}$ & HLL-002 & 4.2 \\
\hline Lilii Bulbus & $\begin{array}{c}\text { Lilium lancifolium } \\
\text { Thunb. }\end{array}$ & bulb & $\begin{array}{l}\text { Nourish Yin and } \\
\text { Moistening the lung }\end{array}$ & HLL-003 & 9.5 \\
\hline Adenophorae Radix & $\begin{array}{l}\text { Adenophora triphylla } \\
\text { (Thunb.) A.DC. }\end{array}$ & root & $\begin{array}{l}\text { Nourish Yin and } \\
\text { Moistening the lung }\end{array}$ & HLL-004 & 20.9 \\
\hline $\begin{array}{l}\text { Asteris Radix et } \\
\text { Rhizoma }\end{array}$ & Aster tataricus L.f. & root & $\begin{array}{l}\text { Moisten the lung to } \\
\text { suppress cough }\end{array}$ & HLL-005 & 25.9 \\
\hline Scrophulariae Radix & $\begin{array}{c}\text { Scrophularia } \\
\text { buergeriana Miq. }\end{array}$ & root & $\begin{array}{l}\text { Nourish Yin and } \\
\text { clear lung fire }\end{array}$ & HLL-006 & 13.1 \\
\hline
\end{tabular}

* Sample specimens and extracts were stored in the Department of Herbal Pharmacology, College of Korean Medicine Kyung Hee University.

\subsection{PF Mouse Model Induction and Sample Administration}

All animal experimental protocols were performed according to the guidance of Kyung Hee University Institutional Animal Care and Use Committee (KHUASP(SE)-18-177). Male ICR mice (7 weeks old, 31-33 g, DaeHan BioLink, $n=6$ ) were used for the study. They were housed for 7 days before induction on a $12 \mathrm{~h}$ light/dark cycle with food and water supplied ad libitum. The animal model was induced using a modified method described in a previous study [14]. The mice were anesthetized using $2 \%$ isoflurane and administered the intratracheal instillation of bleomycin sulfate 
( $2 \mathrm{mg} / \mathrm{kg}, 100 \mu \mathrm{L}$ in sterile saline Dong-A Pharmaceutical Co., Seoul, Korea) for the induction of PF. The mice were randomly divided into 9 groups: (1) normal group, healthy mice without instillation and administration; (2) sham group, mice received sterile saline instillation without administration; (3) control group, mice received bleomycin instillation and distilled water (DW) administration; (4) six treatment groups (TUF, TRS, LIB, ADR, ASR, and SCR), mice received bleomycin instillation and sample administration $(300 \mathrm{mg} / \mathrm{kg})$. All samples were dissolved in DW and administered orally at a volume of $3.3 \mathrm{~mL} / \mathrm{kg}$ simultaneously with lung fibrosis induction at first and then continued twice a day for 10 days. The sham and control groups were given the same volume of DW. Body weight was measured every day at 09:00 for 10 days.

\subsection{Histological Analysis}

The lung was isolated on the 11th day after induction and fixed with $4 \%$ paraformaldehyde. Lung slices (5- $\mu \mathrm{m}$ thick) were stained with hematoxylin and eosin (H\&E) and Masson's trichrome to measure histological changes. Ashcroft scales were measured by two researchers, blinded to the experimental groups according to previous studies [16,17]. Collagen fiber area (\%) of left lung was measured using ImageJ software $(1.43 \mathrm{u}, \mathrm{NIH})$ according to a previous study [18].

\subsection{Broncho Alveolar Lavage Fluid (BALF) Isolation and Immune Cell Count}

BALF was obtained by infusing the lungs three times with $0.5 \mathrm{~mL}$ of cold phosphate-buffered saline ( $\mathrm{pH} 7.2)$ and recovering it using a 20-gauge catheter. The fluid recovery rate was more than $85 \%$. The total cell count was measured using a hemocytometer after methylene blue staining. After confirming that the cells were collected on the slide, H\&E-stained total cells, macrophages, lymphocytes, neutrophils, and eosinophils were counted in a double-blind manner using a microscope (200x).

\subsection{Statistical Analysis}

All the results are presented as mean \pm SEM. A one-way ANOVA followed by a Dunnett's test using GraphPad Prism v.5.0 (GraphPad Software) was used to compare each group to the control group.

\section{Results and Discussion}

The body weight of mice in PF groups decreased immediately after induction while that of mice in normal and sham groups increased continuously. Compared to the control group, all sample treated groups showed the tendency of body weight recovery, but the recovery was not significant (Figure 1A).

The bleomycin-treated control group showed a marked-thickening of the alveolar wall, collapse of alveolar, deposition of inflammatory cells, fibrotic changes (visualized by staining lung sections with H\&E), and collagen deposition (visualized by staining lung sections with Masson trichrome). All sample-treated groups showed a decreased damage score; especially, LIB and ADR showed a significant decrease on the Ashcroft scale compared to the control group $(p<0.05,<0.01)$ (Figure 1B-D).

Collagen fiber area of left lung also showed similar result with that of Ashcroft scale (Figure 1F). LIB and ADR also showed a significant decrease on area of collagen fiber compared to the control group $(p<0.05,<0.01)$ (Figure 1A,F).

Eleven days after lung fibrosis, the number of total cells, macrophages, and lymphocytes in BALF of the control group increased compared to that in normal and sham groups $(p<0.001)$. All sample-treated groups showed decreased cell numbers in BALF compared to the control group. A significant decrease in total cell number in BALF was observed with LIB, ADR, TRS, and SCR compared to the control group $(p<0.05,<0.01$, and $<0.001)$ (Figure 2B). Moreover, a significant decrease in macrophage number in BALF was observed with LIB, ADR, and SCR compared to the control group $(p<0.05$, and $<0.001)$ (Figure 2C). However, only LIB showed a significant decrease in lymphocyte number in BALF compared to the control group $(p<0.01$ and $<0.001)$ (Figure 2D). 
A

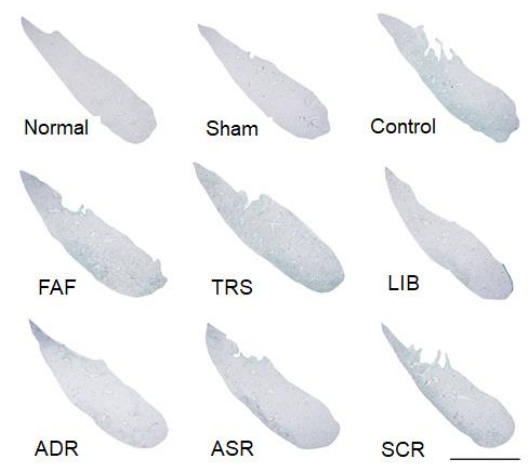

C

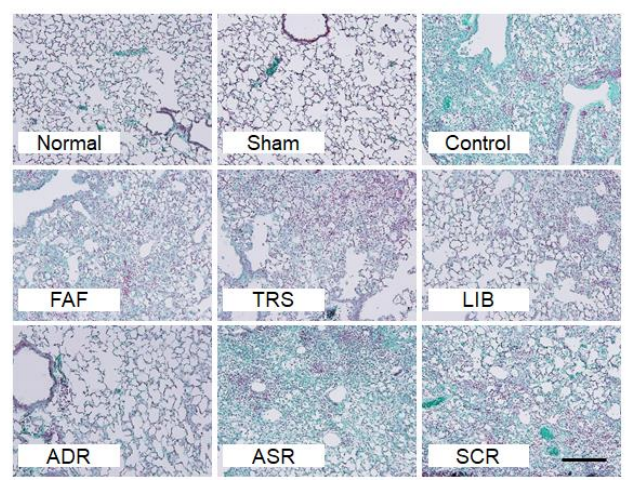

E

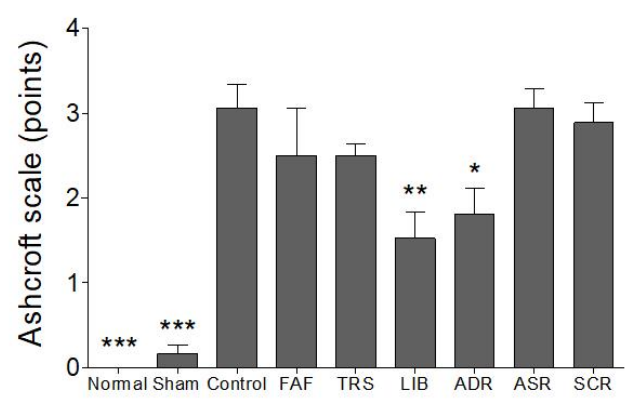

B

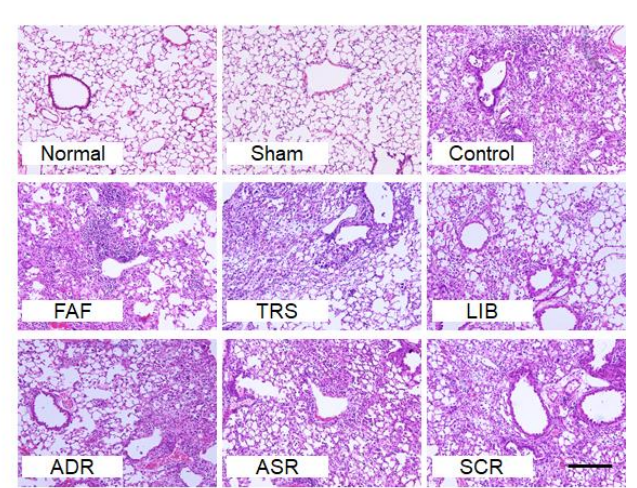

D

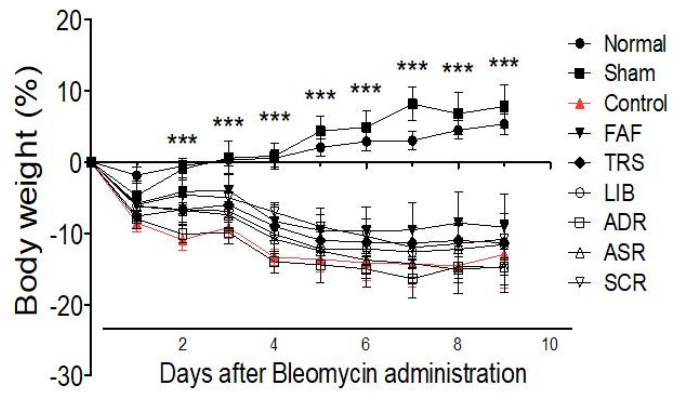

$\mathrm{F}$

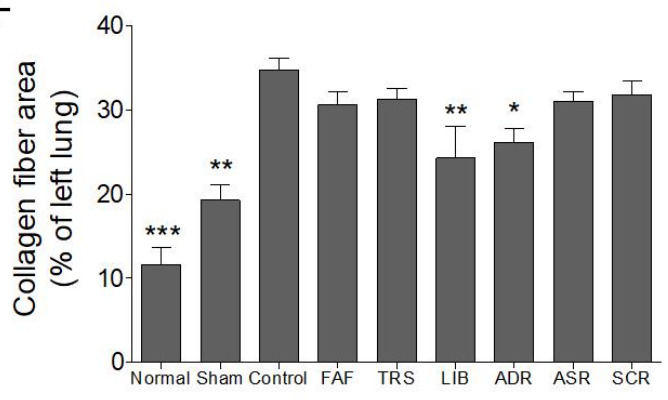

Figure 1. Effects of herbal medicines used to treat pulmonary fibrosis on Masson's trichrome-stained left lung $(10 \times$, A), Hematoxylin-Eosin-stained lung sections $(200 \times, \mathbf{B})$, Masson's trichrome-stained lung sections (200×, C), body weight (D), Ashcroft score (E), and collagen fiber area of left lung (F). Scale bars are $1 \mathrm{~mm}(\mathbf{A})$ and $400 \mu \mathrm{m}(\mathbf{B}, \mathbf{C})$, respectively. FAF, Farfarae Flos; TRS, Trichosanthis Semen; LIB, Lilii Bulbus; ADR, Adenophorae Radix; ASR, Asteris Radix; SCR, Scrophulariae Radix. * represents statistical differences from control group $\left({ }^{*} p<0.05,{ }^{* *} p<0.01,{ }^{* * *} p<0.001\right)$.

In this study, LIB and ADR showed significant effects on the Ashcroft scores in PF mouse model. However, other samples did not show significant effects on fibrotic change. Bleomycin, an anti-cancer agent, is known to be one of the most used inducers of PF animal model with the advantage of short-term PF [14]. The pathogenesis of PF induced by bleomycin is reported to show inflammation between 7 and 9 days and then progress to pulmonary parenchyma and interstitial fibrosis [19]. In this study, LIB and ADR reduced scores on the Ashcroft scale, which is frequently used to evaluate the extent of fibrosis on a scale of 0 to 8 in randomly chosen sections regardless of the staining tool (Masson's trichrome or H\&E) used [20]. 
A

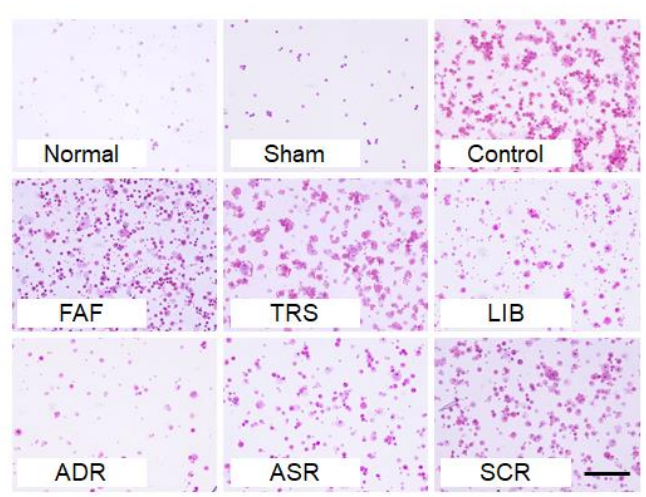

C



B

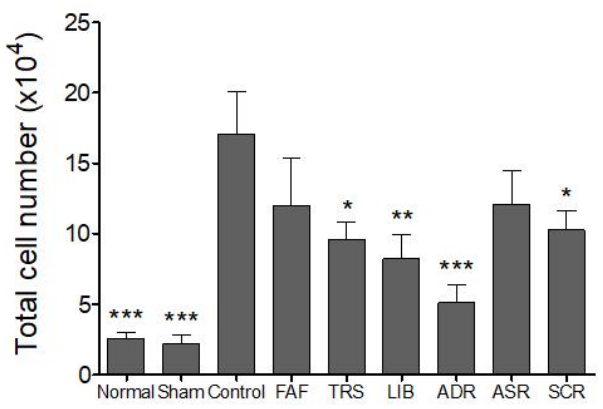

D

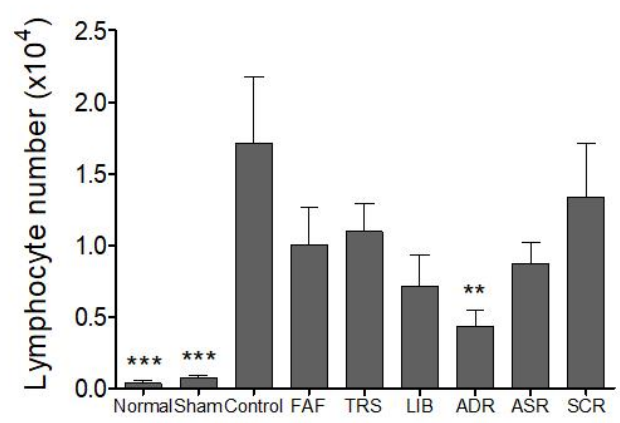

Figure 2. Inflammatory cell counts in the Bronchoalveolar fluid of each group. The typical image of inflammatory cell distribution in each group (A), the graph of the total cell number (B), macrophage number (C), and lymphocyte number (D), respectively. The magnification of A is $200 \times$. Scale bar is $400 \mu \mathrm{m}$. FAF, Farfarae Flos; TRS, Trichosanthis Semen; LIB, Lilii Bulbus; ADR, Adenophorae Radix; ASR, Asteris Radix; SCR, Scrophulariae Radix. * represents statistical differences from control group $\left({ }^{*} p<0.05,{ }^{* *} p<0.01,{ }^{* * *} p<0.001\right)$.

Moreover, in the present study, all sample treated groups showed a tendency to decrease immune cells in BALF. TRS, LIB, ADR, and SCR decreased total cell number; LIB, ADR, and SCR decreased macrophages; and LIB decreased lymphocytes. PF due to bleomycin is most clinically similar to IPF and has the advantage of inducing short-term PF in animal studies [14]. In PF, inflammation is the predominant factor that leads to fibrosis. Alveolar inflammation related to alveolar macrophages, neutrophils, lymphocytes, and other inflammatory cells of the alveoli is known to precede fibrosis of the lung parenchyma and interstitial tissue [19]. Thus, the medicines that decrease the inflammatory cells in BALF might be closely related to the inhibition of fibrotic change in the lung.

In traditional medicine in Korea, China, and Japan, there is no concept of the IPF disease. However, PF characterized with dry cough and dyspnea could be translated to Lung Yin deficiency syndrome, which is also characterized by similar symptoms according to disease identification and treatment in the traditional medicine of these countries [9-11]. Thus, herbal medicines used in the current study have Yin-nourishing and lung-moistening effects that showed the tendency of ameliorating bleomycin-induced PF. Especially, LIB and ADR showed significant inhibitory effects on the fibrotic change in the lung.

Taken together, LIB and ADR could be the major choice for the treatment of the symptoms in PF. However, further studies are needed to investigate the therapeutic range, the maximal effective dosage, and mechanisms of action.

Author Contributions: Conceptualization, J.-W.P., Y.B., J.A., and B.L.; formal analysis; H.J., J.P., K.-I.K., and H.-J.J.; writing-original draft preparation, B.-J.L., H.J., J.A., and Y.B.; writing-review and editing; B.-J.L., H.J. and Y.B. All authors have read and agreed to the published version of the manuscript. 
Funding: This research was funded by a grant of Basic Science Research Program (NRF-2018R1A2B6009621) of the National Research Foundation of Korea (NRF) Funded by the ministry of Science and ICT.

Conflicts of Interest: The authors declare no conflict of interest.

\section{References}

1. Richeldi, L.; Collard, H.R.; Jones, M.G. Idiopathic pulmonary fibrosis. Lancet 2017, 389, 1941-1952. [CrossRef]

2. Raghu, G.; Remy-Jardin, M.; Myers, J.L.; Richeldi, L.; Ryerson, C.J.; Lederer, D.J.; Behr, J.; Cottin, V.; Danoff, S.K.; Morell, F.; et al. Diagnosis of Idiopathic Pulmonary Fibrosis. An Official ATS/ERS/JRS/ALAT Clinical Practice Guideline. Am. J. Respir. Crit. Care Med. 2018, 198, e44-e68. [CrossRef] [PubMed]

3. Kato, E.; Takayanagi, N.; Takaku, Y.; Kagiyama, N.; Kanauchi, T.; Ishiguro, T.; Sugita, Y. Incidence and predictive factors of lung cancer in patients with idiopathic pulmonary fibrosis. ERJ Open Res. 2018, 4. [CrossRef] [PubMed]

4. Vancheri, C.; Failla, M.; Crimi, N.; Raghu, G. Idiopathic pulmonary fibrosis: A disease with similarities and links to cancer biology. Eur. Respir. J. 2010, 35, 496-504. [CrossRef] [PubMed]

5. Abid, S.H.; Malhotra, V.; Perry, M.C. Radiation-induced and chemotherapy-induced pulmonary injury. Curr. Opin. Oncol. 2001, 13, 242-248. [CrossRef] [PubMed]

6. Ballester, B.; Milara, J.; Cortijo, J. Idiopathic Pulmonary Fibrosis and Lung Cancer: Mechanisms and Molecular Targets. Int. J. Mol. Sci. 2019, 20, 593. [CrossRef] [PubMed]

7. Raghu, G.; Collard, H.R.; Egan, J.J.; Martinez, F.J.; Behr, J.; Brown, K.K.; Colby, T.V.; Cordier, J.F.; Flaherty, K.R.; Lasky, J.A.; et al. An official ATS/ERS/JRS/ALAT statement: Idiopathic pulmonary fibrosis: Evidence-based guidelines for diagnosis and management. Am. J. Respir. Crit. Care Med. 2011, 183, 788-824. [CrossRef] [PubMed]

8. Raghu, G.; Rochwerg, B.; Zhang, Y.; Garcia, C.A.; Azuma, A.; Behr, J.; Brozek, J.L.; Collard, H.R.; Cunningham, W.; Homma, S.; et al. An Official ATS/ERS/JRS/ALAT Clinical Practice Guideline: Treatment of Idiopathic Pulmonary Fibrosis. An Update of the 2011 Clinical Practice Guideline. Am. J. Respir. Crit. Care Med. 2015, 192, e3-e19. [CrossRef] [PubMed]

9. Jung, S.K.; Jung, H.J.; Lee, B.J.; Choi, J.Y.; Park, Y.C.; Kim, K.T.; Kim, J.D.; Choi, H.Y.; Suh, W.G.; Park, D.I.; et al. Internal Medicine Pulmonary System; Nado: Seoul, Korea, 2011; pp. 510-511.

10. Shunan, Z.; Lanquan, L.; Hongchun, Z. Clinical Study on Treating Idiopathic Pulmonary Fibrosis with TCM Method of Invigorating Qi, Moisturizing the Lung and Removing Blood Stasis and Toxic Substance. J. Beijing Univ. Tradit. Chin. Med. 1999, 3, 58-61.

11. Zhang, S.; Wu, H.; Liu, J.; Gu, H.; Li, X.; Zhang, T. Medication regularity of pulmonary fibrosis treatment by contemporary traditional Chinese medicine experts based on data mining. J. Thorac. Dis. 2018, 10, 1775-1787. [CrossRef] [PubMed]

12. Chen, M.J.; Yang, G.L.; Ding, Y.X.; Tong, Z.Q. Efficacy of TCM therapy of tonifying lung-kidney's Qi-deficiency in a case of idiopathic pulmonary fibrosis: A case report. Medicine (Baltimore) 2019, 98, e15140. [CrossRef] [PubMed]

13. Shi, X.; Qu, N.; Pang, L.; Liu, C. Discussion on the pathogenesis of "Yin deficiency of lung-kidney and Phlegm in meridian" in idiopathic pulmonary fibrosis. Jilin J. Tradit. Chin. Med. 2014, 34, $220-222$.

14. Moore, B.B.; Hogaboam, C.M. Murine models of pulmonary fibrosis. Am. J. Physiol. Lung Cell Mol. Physiol. 2008, 294, L152-L160. [CrossRef] [PubMed]

15. Zhonghua Bencao Edit Committee. Pharmacopoeia of the People's Republic of China; Zhonghua Bencao Edit Committee: Shanghai, China, 2015.

16. Ashcroft, T.; Simpson, J.M.; Timbrell, V. Simple method of estimating severity of pulmonary fibrosis on a numerical scale. J. Clin. Pathol. 1988, 41,467-470. [CrossRef] [PubMed]

17. Zhou, X.M.; Wen, G.Y.; Zhao, Y.; Liu, Y.M.; Li, J.X. Inhibitory effects of alkaline extract of Citrus reticulata on pulmonary fibrosis. J. Ethnopharmacol. 2013, 146, 372-378. [CrossRef] [PubMed]

18. Majeed, H.; Okoro, C.; Kajdacsy-Balla, A.; Toussaint, K.C., Jr.; Popescu, G. Quantifying collagen fiber orientation in breast cancer using quantitative phase imaging. J. Biomed. Opt. 2017, 22. [CrossRef] [PubMed] 
19. Williamson, J.D.; Sadofsky, L.R.; Hart, S.P. The pathogenesis of bleomycin-induced lung injury in animals and its applicability to human idiopathic pulmonary fibrosis. Exp. Lung Res. 2015, 41, 57-73. [CrossRef] [PubMed]

20. Mouratis, M.A.; Aidinis, V. Modeling pulmonary fibrosis with bleomycin. Curr. Opin. Pulm. Med. 2011, 17, 355-361. [CrossRef] [PubMed]

(C) 2020 by the authors. Licensee MDPI, Basel, Switzerland. This article is an open access article distributed under the terms and conditions of the Creative Commons Attribution (CC BY) license (http://creativecommons.org/licenses/by/4.0/). 